\title{
Investigation and Analysis of Current College Students' Attitudes to Artificial Intelligence
}

\author{
Ma Lan \\ Marxism School, Jianghan University, Wuhan 430056,China \\ lanhorse@163.com
}

Keywords: Artificial Intelligence, emerging technology, future prospects

\begin{abstract}
Artificial Intelligence (AI) is a rapidly developing and widely used technology today. The same as other new technology, it will enlarge people's new knowledge about emerging technology. Now more and more scientists and philosophers pay attention to the influence on human society of AI. Over the discussion of AI, the views of college students that have been largely neglected from the discussion. We need interdisciplinary discussion, especially if we seek to the public policy of AI in the future in China. The data collected suggest that not all the college students nowadays in China are interested in the AI products and are optimistic to the development of AI. Taken together, the investigation data might be helpful to assess various ethical arguments which are for and against AI.
\end{abstract}

\section{Introduction}

Artificial intelligence (AI), sometimes called machine intelligence, is intelligence demonstrated by machines, in contrast to the natural intelligence displayed by humans and other animals. [1]At the 2017 Future of Go Summit, AlphaGo won Ke Jie, the world No.1 ranked weiqi player in the world. AlphaGo is a computer program that plays the board game Go, which is not the first time to beat top players. These competitions aroused people's focus on AI ability and more and more media report begin to worry it will challenge human traditional value views. In fact, it is undeniable that AI products have begun to enter the people's life, such as sweeping robot and intelligent nanny. Although they are not as horrible as the description in science fiction films, once out of reach of AI is indisputable fact that concepts are a reality. The current situation of AI, however it will develop, is a topic of great concern to the academic community. They have proposed some ethical disputes and social issues in professional and academic field, such as unemployment, unsafety, interest and benefit. AI of research and applications is a multidisciplinary and multi-faceted area.

\section{Experimental Methods and contents}

To ensure the effective data for the samples, we have interviewed 10 college students including 25 male college students and 15 female college students with ages from 19 to 22 years living in different universities in different cities in China, and from different major background of history, literature, computer, and electronic information and different family in May,2018. Of course, they were presented 
with a brief demographic questionnaire though e-mail and they tended to accept this kind of questionnaire survey. Participants are provided informed consent and we used a semi-structure design, with a list of open questions. Additionally, we give different optional context for participants to explain the reason behind their choices. To confirm the participants fully understand the context of the survey, we have a communication through telephone to ensure their understand capacities. The contents what we design in the survey and the analysis results are as follows:

Understanding the concept and development situation of AI:

AI is a hot topic in the public. From table 1 we know 26 college students of 52\% participants understand what is AI. And 24 college students of $48 \%$ don't know the exactly the concept of AI. Of course, some students don't know exactly about the present situation of AI development. 18 students of the 50 participants focus on the development of AI. 32 students of $64 \%$ participants don't care about the future development prospect of AI and they don't use any AI products. The investigation data is showed as below:

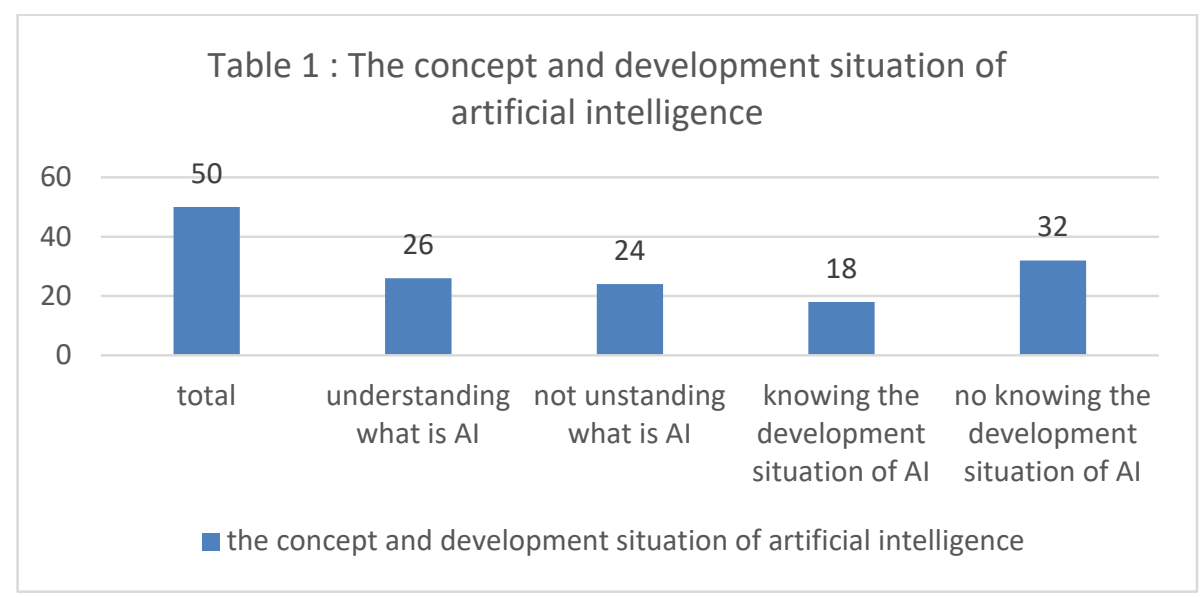

Attitude of college students to artificial intelligence:

From the investigation table 2, nearly fifty percent of the participants don't agree the view that AI has replace human intelligence. But more experts in AI field believe that AI can surpass humans through technology progress. A bit more students think AI will do all the things human can through continuous study. 18 students of the $36 \%$ participants are conservative about the limits of AI and they AI will destroy human society ethical regulation. 32 of the 50 participants take positive attitude and they hope AI could help human beings to deal with all kinds of staff task in the future. Only a small number of students worry about the development of artificial AI will pose a threat to humans. Though AlphaGo beat all the smart weiqi players, they have the confident that human beings can develop new regulation and capability to control AI.

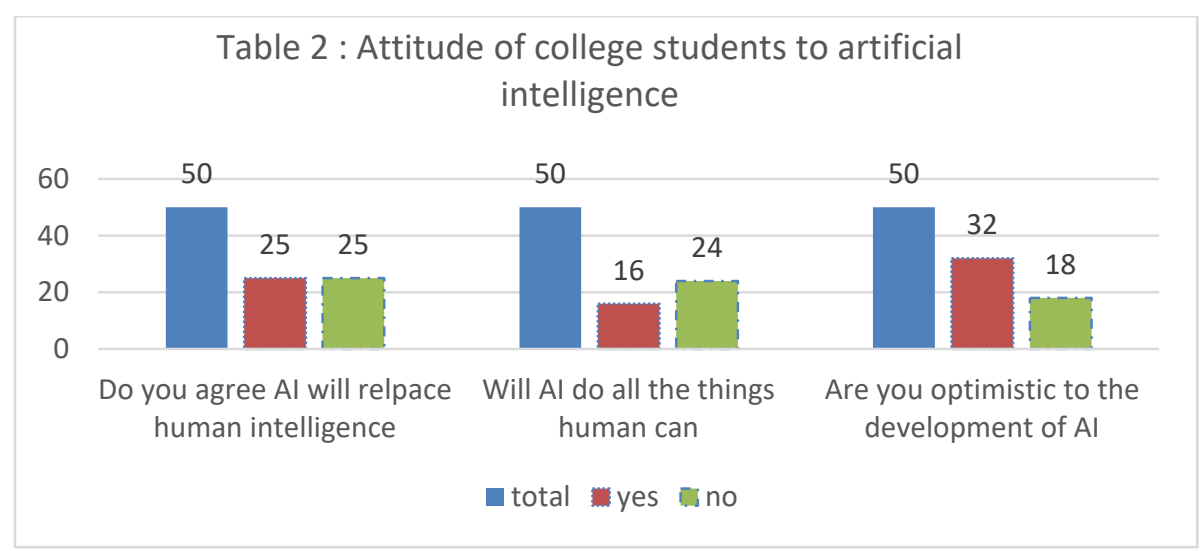


The amount and the reasons the participants against AI:

Table 3 shows 20 college students of $40 \%$ participants are against developing AI. AI will cause ethical issues such as unemployment, violating the humanity and unsafety . 8 students of 20 against AI think AI provides automatic working method and it will make people lose their jobs. Artificially intelligent bots are becoming better and better at modelling human conversation and relationships, but they are not human beings. 5 participants think it is unnatural and violating the humanity. 7 participants think unsafety is another reason which should be taken into account. The more powerful a technology becomes, the more can it be used for nefarious reasons as well as good. AI robots produced to replace human soldiers, or autonomous weapons, but to AI systems that can cause damage if used maliciously.

Table 3: The amount and the reasons participants against

Al

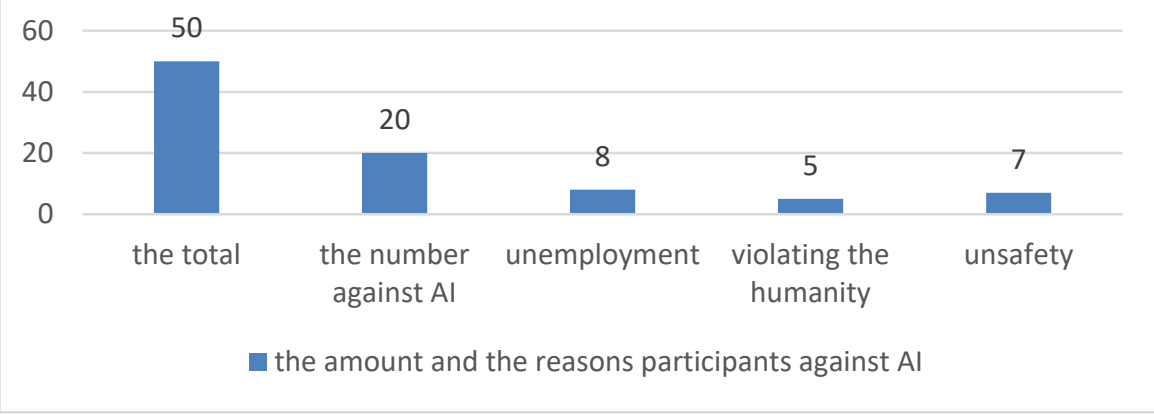

The amount and the reasons the participants for AI:

30 participants of $60 \%$ participants are for AI. Robots can work, study, do housework and even help care for the elderly. In this respect, the development of AI is of great benefit to human beings. there are many profits in the market for companies if they sell AI products. Moreover, scientists have the interest and right to do research in the field. Table 4 lists the reasons and amount the participants are for the AI.

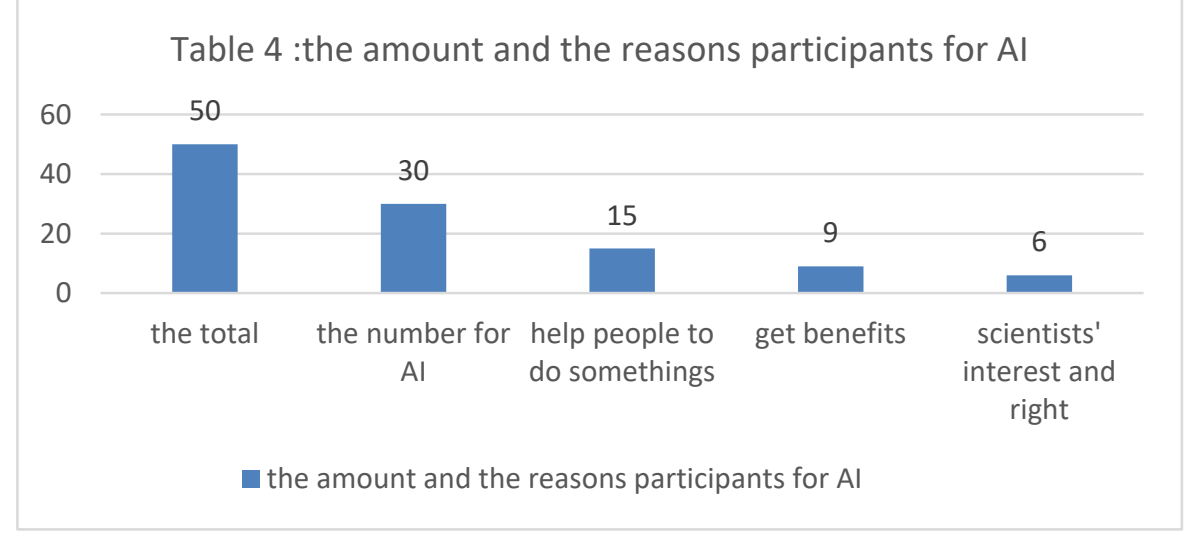

\section{Summary}

Most students understand AI through mass media, and a few students understand artificial intelligence in professional fields. Mass media has contributed to the concept of artificial intelligence and the publicity and popularization of relevant information. However, less publicity or education related to artificial intelligence knowledge are provided in schools. Those who have used AI products focus on it. On the contrary, those don't pay attention to AI products because they don't contact any AI products.

AI researchers and scientist may have good reason for their reluctance to wade into ethics. The questions raised are likely to be open-ended, and their research both inside and outside the laboratory may be supported by some projects. Over the discussion of AI, it is necessary to analyze the attitudes of the 
college students in China. College students are no longer the traditional image of reading in the "ivory tower", Moreover, they are becoming more integrated into the society. College students' attitude represent a future market direction that can be assess and explore AI development. In modern life, college students' knowledge of AI is very important. We collected relevant data to investigate the attitude of college students to AI and current college students' understanding of AI. Of course, from the data we also consider the reasons participants against or for the AI. Correspondingly, we can provide reference suggestions and directions for AI developers from the investigation data.

\section{References}

[1] Information on https://en.wikipedia.org/wiki/Artificial_intelligence.

[2] Wan Genxun, Li Jianyong, Qiang Tian, et al. Test system and test method of millions of one-to-many face recognition products:, CN 101615196 A[P]. 2009.

[3] Hwang W, Kee S, Park G, et al. Face recognition method, and system using gender information: US, US 20070104362 A1[P]. 2007.

[4] Phillips P J, Flynn P J, Scruggs T, et al. Overview of the Face Recognition Grand Challenge[C]// IEEE Computer Society Conference on Computer Vision and Pattern Recognition. IEEE Computer Society, 2005:947-954.

[5] Keers R N, Williams S D, Cooke J, et al. An Overview of Holistic Face Recognition [J]. ljrcct, 2013, 2(9):738-741. 